Zum Abschluss der Tagung am Freitag richteten Dr. Kerstin Niethammer-Jürgens (Rechtsanwältin, Fachanwältin Familienrecht, Potsdam) sowie Renate Maltry (Rechtsanwältin, Fachanwältin Erbrecht und Fachanwältin Familienrecht, München) den Blick nach Brüssel. Im Scheidungsrecht gilt es aufgrund der Rom-III-Verordnung seit Juni 2012 zu beachten, dass nicht mehr die Staatsangehörigkeit entscheidend dafür ist, nach welchem europäischen Recht Eheleute geschieden werden können, sondern vielmehr deren gewöhnlicher Aufenthalt. Auch im Erbrecht werden beginnend ab dem Jahr 2015 mit der Erbrechtsverordnung einheitliche Regeln darüber entscheiden, welches Erbrecht auf einen intereuropäischen Erbfall anzuwenden ist.
Der Informations- und Beratungsbedarf der Bevölkerung wird aufgrund der europarechtlichen Einflüsse im Familien- und Erbrecht steigen.

Allen Rednerinnen und Rednern danken wir herzlich für ihre ausgezeichneten und beachtenswerten Beiträge. Eröffnungsrede, Grußworte und Festvortrag sind in diesem Heft dokumentiert. Die familienrechtlichen Vorträge von Dr. Isabell Götz, Prof. Dr. Barbara Dauner-Lieb, Gretel Diehl, Dr. Kerstin NiethammerJürgens und Renate Maltry folgen in Heft 1/2014.

Der Kongress wurde vom Bundesministerium für Familie, Senioren, Frauen und Jugend (BMFSFJ) finanziell unterstützt. Auch dafür bedanken wir uns.

\title{
Eröffnung des 40. Bundeskongresses des Deutschen Juristinnenbundes am 26. September 2013 in Leipzig*
}

\section{Ramona Pisal \\ Präsidentin Deutscher Juristinnenbund, Berlin}

Sehr geehrte Frau Eckertz-Höfer, sehr geehrte Frau Dr. Grundmann, sehr geehrte Frau Munz, sehr geehrter Herr Müller, sehr geehrte Damen und Herren, liebe Kolleginnen im Deutschen Juristinnenbund,

Es ist mir eine große Freude und besondere Ehre, Sie alle, Mitglieder und Gäste, heute Abend hier im prächtigen Gebäude des Bundesverwaltungsgerichtes in Leipzig zur Eröffnung des 40. Bundeskongresses des Deutschen Juristinnenbundes begrüßen zu dürfen.

Den musikalischen Auftakt - wie könnte es in dieser Stadt anders sein: selbstverständlich mit Bach - verdanken wir der Violinistin Franziska Hoelscher und ihrer Violine, deren Kunst wir im Laufe des Abends noch einige Male genießen dürfen.

Ihnen, liebe Frau Eckertz-Höfer, gilt unser besonderer Dank dafür, dass Sie uns als Präsidentin des Bundesverwaltungsgerichts Ihr denkmalgeschütztes Haus für diesen festlichen Abend, die Fachtagung und die Mitgliederversammlung am Samstag öffnen und außerdem so liebenswürdig waren, die Festrede zu übernehmen. Wir freuen uns sehr, dass wir bei Ihnen zu Gast sein dürfen und wissen diese besondere Atmosphäre auch besonders zu schätzen.

Sicher haben viele von Ihnen bereits die Gelegenheit genutzt, heute Nachmittag im Rahmen sachkundiger Führung mehr über dieses prächtige Haus und seine wechselvolle, nicht immer unbelastete Geschichte zu erfahren. Es ist ein großes Glück, dass dieser eindrucksvolle Justizpalast, einer der größten und imposantesten in Europa, die Zeitläufte überstanden hat und heute einerseits als Denkmal, andererseits als lebendiger Ort der Rechtsanwendung und Rechtsfindung den Wert von Rechtsstaatlichkeit repräsentiert und deren Kontinuität anmahnt. Dass dieses Haus nicht von der Figur der Gerechtigkeit gekrönt wird wie sonst oft, sondern die Wahrheit buchstäblich ganz oben angesiedelt ist, gehört für mich zu den bemerkenswerten Details dieses beeindruckenden Bauwerks.

Wir freuen uns sehr, dass der 1. Bürgermeister der Stadt Leipzig heute Abend bei uns ist und ich begrüße herzlich Herrn Andreas Müller. Haben Sie bereits jetzt vielen Dank für das Grußwort, das Sie gleich an uns richten werden.

Nur vier Tage nach der Bundestagswahl war dieser Termin für unsere politisch engagierten Gäste besonders schwer einzuhalten.

Als Vertreterin der Exekutive erwarten wir Frau Staatssekretärin im Bundesministerium der Justiz, Frau Dr. Grundmann, die später ein Grußwort zu uns sprechen wird.

Wir freuen uns sehr über die Anwesenheit der Präsidentin des Sächsischen Verfassungsgerichtshofs, Frau Birgit Munz, außerdem Vizepräsidentin des Oberlandesgerichts Dresden. Auch Ihnen danke ich herzlich für Ihre Bereitschaft, ein Grußwort zu uns zu sprechen.

Wir freuen uns, dass die Präsidentin des Bundesarbeitsgerichts, Frau Ingrid Schmidt, auch heute Abend bei uns sein kann. Vor sechs Jahren haben wir in Erfurt getagt und wir erinnern uns sehr gerne an den Abend im Bundesarbeitsgericht, liebe Frau Schmidt.

Als weitere Repräsentanten und Repräsentantinnen der 3. Gewalt begrüße ich die Bundesrichterinnen Frau Friederike Grube, Frau Dr. Gabriele Hessel, Frau Christine MeßbacherHönsch, Frau Dr. Renate Philipp, Frau Dr. Ursula Schneider, Frau Maren Thomsen, Frau Dr. Regine Winter und den Bundesrichter Herrn Dr. Martin Fleuß, Richter am Bundesverwaltungsgericht und gleichzeitig 2. stellvertretender Vorsitzender des Bundes Deutscher Verwaltungsrichter und Verwaltungsrichterinnen.

Es mögen kurz entschlossen weitere Richterinnen und Richter gekommen sein, die wir nicht auf der Teilnehmerliste haben, Sie alle sind uns herzlich willkommen.

alle Fotos: Katrin Lange, djb 
Sieben Bundesrichterinnen, ein Bundesrichter - schließen Sie bitte aus diesem Verhältnis 7:1 nicht auf einen entsprechenden Frauenanteil an der Bundesrichterschaft. Der djb ist zwar sehr erfolgreich mit seinem von Vizepräsidentin Eva Schübel initiierten Projekt „Frauen in die Roten Roben“, das zu deutlich mehr Wahlvorschlägen für Richterinnen geführt hat. Der Richterwahlausschuss hat in diesem Jahr zum ersten Mal in seiner Geschichte für den Bundesgerichtshof mehrheitlich Frauen gewählt: Sechs von acht Stellen wurden mit Frauen besetzt. Aber das wird noch oft passieren müssen, wenn wir die deutliche Unterrepräsentanz von Richterinnen an den Bundesgerichten nachhaltig verändern wollen. Ob hier oder in der Wirtschaft: Die Frauen sind da. Sie müssen nur in den Blick genommen und angesprochen werden.

Auch darum herzlichen Dank, Herr Dr. Fleuß, dass Ihr Verband die Kolleginnen bereits im Namen explizit benennt.

Das ist alles andere als selbstverständlich. Meist werden die Frauen mittels Fußnote darauf verwiesen, sich über einen mit * markierten Hinweis „mitgemeint“ zu fühlen, wenn ausdrücklich nur die männliche Form, das generische maskulinum, genannt wird. Dass sich dieses Gefühl aber so leicht nicht einstellt, wird aktuell ganz empfindlich registriert, seitdem in der Satzung der Universität Leipzig umgekehrt verfahren wird. Dort dürfen sich nämlich jetzt die Herren „mit angesprochen“ fühlen, wenn von Professorinnen die Rede ist. Diese Initiative ist in den Medien stark diffamiert worden; tatsächlich wird dort kein Professor als Professorin angesprochen und kein Mann als Frau, nur die Satzung wurde entsprechend gefasst und mit dem berühmten * versehen, noch dazu aus gutem Grund: 60 Prozent der Studierenden und 53 Prozent der Promovierten an der Leipziger Hochschule sind weiblich - keine schlechte Bilanz für uns Frauen. Die mediale Empörung zeigt die Kränkung derer, die sich durch das * nicht mitgemeint, sondern ausgeblendet, ignoriert fühlen. Das können wir gut verstehen. Eine heilsame Lehre, so hoffen wir, die auch insoweit von der Universität Leipzig ausgehen mag, und bedanken uns herzlich bei der Rektorin Frau Professorin Schücking für diesen Weckruf, dem andere bereits folgen. Der $\mathrm{djb}$ ist konservativ und macht es wie immer. Wir sprechen beide an. Dass ich eben nur die Kolleginnen im djb begrüßt habe, liegt daran, dass es Kollegen im Verband nicht gibt, nicht einmal als fördernde Mitglieder.

Wir freuen uns über die zahlreichen Gäste aus der Region, den Präsidenten des Landgerichts Leipzig, Herrn Karl Schreiner, die Präsidentin des Landgerichts Magdeburg, Frau Sigrid Jaspers, den Präsidenten des Amtsgerichts Magdeburg, Herrn Uwe Wegehaupt, die Vizepräsidentin des Amtsgerichts Dresden, Frau Stephanie Vossen-Kempkens, und die Direktorin des Amtsgerichts Wittenberg, Frau Siegrun Baumgarten.

Weiter geben uns die Ehre der Präsident der Bundesrechtsanwaltskammer a.D., Rechtsanwalt Dr. Bernhard Dombek, Frau Rechtsanwältin Heike Bruns als Vizepräsidentin der Rechtsanwaltskammer Sachsen, Herr Stephan Kopp, Hauptgeschäftsführer der Rechtsanwaltskammer München, Frau Rechtsanwältin Theresa Nentwig und Frau Rechtsanwältin Dr. Katrin Seime, beide Mitglied des Vorstandes der Rechtsanwaltskammer Thü- ringen, sowie Rechtsanwältin Frau Susanne Drehsen, Mitglied der Rechtsanwaltskammer Bamberg.

Mit besonderer Freude begrüße ich aus dem Deutschen Anwaltverein (DAV) Frau Rechtsanwältin und Notarin Mechtild Düsing, Mitglied des Vorstandes des DAV, Herrn Dr. Cord Brügmann, Hauptgeschäftsführer des DAV, und Frau Rechtsanwältin Eva Becker, Vorsitzende des Geschäftsführenden Ausschusses der Arbeitsgemeinschaft Familienrecht im DAV sowie Frau Rechtsanwältin Silvia Groppler, Vorsitzende der Arbeitsgemeinschaft Anwältinnen im DAV.

Ein herzliches Willkommen auch Frau Rechtsanwältin Annett Seifert, Vorstandsvorsitzende des Zwickauer Anwaltvereins, Herrn Rechtsanwalt Dr. Daniel Fingerle, Vorsitzender des Leipziger Anwaltvereins und Frau Rechtsanwältin Cornelia Lotzmann, Vorsitzende des Halleschen Anwaltvereins.

Für die Familienrechtlerinnen und Familienrechtler ist dieser September ein einziger Tagungsmarathon. Umso dankbarer bin ich Frau Richterin am Oberlandesgericht Dr. Isabell Götz und Herrn Vorsitzenden Richter am Oberlandesgericht Professor Brudermüller, dass sie vom Deutschen Familiengerichtstag (DFGT), der erst am vergangenen Samstag zu Ende gegangen ist, heute den Weg zu uns nach Leipzig gefunden haben. Ihnen, liebe Frau Dr. Götz, gratuliere ich sehr herzlich zu Ihrer Wahl als neue 1. Vorsitzende des DFGT. Ihnen, lieber Herr Professor Brudermüller, gratuliere ich herzlich zur gelungenen Nachfolgeregelung und wünsche Ihnen nach mehr als zehn Jahren Vorsitz zukünftig entspannten Genuss als Ehrenvorsitzender.

Traditionell pflegen wir unsere Verbindungen $\mathrm{zu}$ anderen Frauenverbänden und Institutionen, die uns nahe stehen. Dazu gehören natürlich der Deutsche Frauenrat, dessen Vorsitzende Frau Hannelore Buls ich sehr herzlich begrüßen darf. Als Mitglied des Bundesvorstandes von ver.di ein herzliches Willkommen an Frau Ministerialdirektorin a.D. Eva-Maria Welskop-Deffaa, an die Direktorin des Deutschen Instituts für Menschenrechte, Frau Professorin Beate Rudolf, die Vorsitzende des Vereins Anwältinnen ohne Grenzen, Frau Jasmina Prpić, die Past Präsidentin des Deutschen Akademikerinnenbundes Frau Professorin de Sotelo und last but not least die Vorsitzende von proQuote Medizin, Frau Professorin Gabriele Kaczmarczyk, unsere Mitstreiterin im Aktionsbündnis Spitzenfrauen, das sie heute Abend gleichzeitig mitrepräsentiert.

Den Reigen der ausdrücklichen Begrüßungen möchte ich schließen mit vier Frauen, die als hoch verdiente Mitglieder unseres Verbandes unsere besondere Wertschätzung genießen. Das sind unsere Ehrenpräsidentinnen, Frau Rechtsanwältin Renate Damm und Frau Antje Sedemund-Treiber, Präsidentin des Bundespatentgerichtes im Ruhestand, sowie Pastpräsidentin Frau Rechtsanwältin und Notarin Jutta Wagner, meine Vorgängerin im Amt. Unsere 3. Ehrenpräsidentin, Frau Senatorin a.D. Rechtsanwältin Dr. Lore Maria Peschel-Gutzeit - sonst uns immer treu - kann heute leider nicht persönlich anwesend sein, aber irgendwie ist sie es doch. Wie genau, das erfahren Sie später.

Im Übrigen ist bei uns Frau Rechtsanwältin Ursula Raue, ebenfalls djb-Präsidentin - damals hieß dies noch etwas weniger spektakulär Vorsitzende - in den Jahren 1993 bis 1997. 
Wir sind sehr gerne zu Gast in Ihrer schönen Stadt, Herr Bürgermeister, denn frauenbewegte Frauen bewegen sich hier auf historischem Pflaster. Dabei denke ich nicht in erster Linie an die Schriftstellerin Hedwig Courths-Mabler, die als nichteheliche Halbwaise früh auf sich gestellt war und in Leipzig als Gesellschafterin und Vorleserin einer alten Dame Geld verdiente, später in Leipzig heiratete und 15 Jahre nach der Familiengründung ihren ersten Roman veröffentlichte. Der trug den Titel: "Licht und Schatten“. Heutzutage, das habe ich letzte Woche in Brühl von Herrn Brudermüller gelernt, werden Ehen im Schnitt nach 14,6 Jahren geschieden. Ob diese Erkenntnis mit dem Titel zu tun hat, weiß ich nicht, aber ihre ungemeine Produktivität und weitere Romane, darunter: „Die wilde Ursula“, „Ich will“, „Eine ungeliebte Frau“, „Die schöne Unbekannte“, „Der Scheingemahl“, „Wenn Wünsche töten könnten“, und letztlich: „Die Flucht vor der Ehe“, könnten schon zu allerlei Spekulationen Anlass geben.

Nein, Courths-Mahler könnte uns nicht nach Leipzig locken, obwohl dieser Frau Respekt zu zollen ist, die bei schlechten Startchancen zielstrebig und erfolgreich ihren Weg gemacht hat, als Gesellschafterin, als Verkäuferin und schließlich als Schriftstellerin, deren Werke noch heute verfilmt werden - 2005 war die letzte Verfilmung.

Es war auch nicht die Hexenküche in Auerbachs Keller, die für uns den besonderen Reiz ausmachte, obwohl wir dort morgen Abend sein werden; allerdings zum Essen, nicht zum Kochen.

Wir fühlen uns aus einem anderen Grunde hier genau am richtigen Ort, denn Leipzig, so sagt es die alma mater lipsiensis anlässlich ihrer 600-Jahrfeier, ganz unverdächtig vier Jahre vor der Umstellung auf das generische femininum, Leipzig gilt als die Wiege der deutschen Frauenbewegung. Leipziger Bürgerinnen gründeten hier 1865 den ersten Frauenverein in Deutschland, den Allgemeinen Deutschen Frauenverein (ADF). Über die Leipziger Frauen hören wir gleich mehr vom Herrn Bürgermeister. Und das Rahmenprogramm bietet Ihnen morgen die Möglichkeit, auf den Spuren dieser starken Frauen zu wandeln, die für uns alle den Boden bereitet haben, und viel mehr über sie und die erste große Frauenbewegung in Deutschland zu erfahren.

Nur eines möchte ich gern erwähnen, weil wir Juristinnen sind und es mir auch nach beinahe 150 Jahren so vertraut und aktuell im Ohr klingt: Das Hauptziel des ADF war die rechtliche Gleichstellung von Mann und Frau sowie ein gleichberechtigter Zugang zu Erwerbsarbeit und Bildung. In Paragraph 1 seiner Satzung heißt es: „Der Allgemeine Deutsche Frauenverein hat die Aufgabe, für die erhöhte Bildung des weiblichen Geschlechts und die Befreiung der weiblichen Arbeit von allen ihrer Entfaltung entgegenstehenden Hindernissen mit vereinten Kräften zu wirken."

Die Vereinszeitschrift „Neue Bahnen“ - also das Gegenstück zur djbZ - sollte daher „dem weiblichen Fortschritt“ dienen und „keine Modebilder, keine Stick- und Schnittmuster, keine Recepte“ enthalten - dies alles fände „sich anderwärts zur Genüge“, meinten die Herausgeberinnen. Sie forderten Industrie- und Handelsschulen für Mädchen, Arbeiterinnenschutz, Mutterschutz, Chancengleichheit im Beruf und - Sie werden es kaum glauben - gleichen Lohn für gleiche Arbeit und Gewerbefreiheit für Frauen (Quelle: Deutsches Historisches Museum).

Die erhöhte Bildung des weiblichen Geschlechts haben die Frauen erreicht: Nie gab es besser ausgebildete Frauen als heutzutage. Sie erinnern sich an die Zahlen der Universität Leipzig. Aber wie steht es mit der Befreiung der weiblichen Arbeit von allen ihrer Entfaltung entgegenstehenden Hindernissen? Chancengleichheit, gleicher Lohn für gleiche Arbeit? Knapp 150 Jahre haben nicht gereicht, diese Ziele zu verwirklichen.

Den Befund muss ich Ihnen nicht schildern, Sie kennen die Themen alle und auch die Zahlen, denn immerhin ist es uns mit vereinten Kräften gelungen, die Frauenpolitik in den Fokus der öffentlichen Wahrnehmung und politischen Diskussion zu rücken. Und dort werden wir sie auch halten und politische Lösungen fordern. Das ist tatsächlich alternativlos, denn die bestehenden Ungleichgewichte und die Verteilung von Lasten zum Nachteil von Frauen sind nicht nur unfair und ungerecht, sie sind unseres demokratisch verfassten Rechtsstaates in der Mitte Europas mit Vorbildfunktion für viele andere Gesellschaften schlichtweg unwürdig. Wie sollen wir den anderen erklären, dass Frauen in Deutschland bis zu 22 Prozent Entgeltunterschiede gegenüber Männern hinnehmen und dass sie im Alter mit einem Drittel der Rente eines Mannes auskommen müssen, dass 90 Prozent der Alleinerziehenden Frauen sind, die zu einem großen Teil mit ihren Kindern in Armut leben. Frau könnte sich fremdschämen, wenn sie sich das nicht längst abgewöhnt hätte. Stattdessen formulieren wir deutlich unsere Erwartungen.

Am 17. Mai 2013 haben wir im Aktionsbündnis „Spitzenfrauen " die Spitzenkandidatinnen und Spitzenkandidaten persönlich aufgesucht, wir haben unsere Wahlprüfsteine formuliert und fordern von der Bundesregierung eine konsequente, nachhaltige und vor allem konsistente Gleichstellungspolitik - ohne Widersprüche. Ihre Aufgabe ist es, Rahmenbedingungen zu schaffen, die es jeder Frau und jedem Mann ermöglichen, gemäß ihren Vorstellungen, Wünschen und Fähigkeiten zu leben, mit der realistischen Chance auf faire Teilhabe in allen gesellschaftlichen Bereichen, in der Familie wie im Beruf. Das wäre dann echte Wahlfreiheit und den Auftrag dazu finden wir im Grundgesetz.

Eigenständige Existenzsicherung für Frauen ist insbesondere nach der Reform des Unterhaltsrechtes unabdingbar und für unsere Arbeit von zentraler Bedeutung. Sie erfordert auskömmliche Erwerbsarbeit, kontinuierlich, im gesamten Lebensverlauf. Das betont auch der 1. Gleichstellungsbericht der Bundesregierung. Dieses Ziel wird konterkariert durch Fehlanreize, vor allem das Ehegattensplitting, dessen Abschaffung wir seit Jahrzehnten fordern. Diese Form der Besteuerung fördert nicht Familien, sie fördert absolut einseitig eine bestimmte Form männlicher Karrieren, sie hält Frauen von existenzsichernder Arbeit fern und kostet ca. 20 Milliarden Euro im Jahr, die alle Steuerzahlerinnen und Steuerzahler aufbringen müssen. Dieselbe Wirkung hat das Betreuungsgeld, das umgehend abgeschafft werden müsste, wie auch die Minijobs, die überwiegend Frauen in völlig unzureichende Beschäftigungsverhältnisse bringen und dort auch halten.

Wir sind für verbindliche Mindestlöhne, als geeignetes Mittel gegen die unerträglich niedrigen Entlohnungen in sogenannten 
frauentypischen Berufen, und für ein Entgeltgleichheitsgesetz, denn der Grundsatz gleicher Lohn für gleiche Arbeit muss nun endlich durchgesetzt werden.

Und wir fordern eine verbindliche Frauenquote für die Führungspositionen in der Privatwirtschaft, damit die Unternehmen endlich auch die weibliche Karriere in den Blick nehmen und die Frauen angemessen fördern, anstatt sie weiterhin als billiges Personal, am liebsten in Teilzeit, unter deren Qualifikation zu beschäftigen. 300 Hauptversammlungen börsennotierter Unternehmen haben wir im Projekt „Aktionärinnen fordern Gleichberechtigung “ in den letzten vier Jahren besucht und nach den Frauen auf allen Karrierestufen gefragt. Tausend Mal haben wir hören müssen, dass die Auswahl allein aufgrund der Qualifikation erfolgt sei. Gut, dass es uns so oft gesagt worden ist, denn angesichts der in der Öffentlichkeit breit diskutierten Fehler und Skandale im Management großer deutscher Unternehmen wären wir darauf von alleine wahrscheinlich nicht gekommen.

Als Juristinnen ist für uns das Familienrecht immer ein besonderer Schwerpunkt. So beschäftigen wir uns morgen während unseres Fachkongresses mit dem Thema Auseinandersetzung und Teilhabe am ehelichen Vermögen. Am Ende geht's ums Geld - das trifft auch und gerade bei Scheidung und im Erbfall zu. Ich freue mich auf einen spannenden Austausch und hoffe, Sie sind dabei.

Der djb wurde 1948 in Dortmund gegründet. Kann die reife Dame djb im 65. Lebensjahr in den wohlverdienten Ruhestand gehen und zufrieden lächelnd auf ihr Lebenswerk zurückblicken, in der ruhigen Gewissheit, dass die Gleichberechtigung der Frau mit den Mitteln des Rechts tatsächlich umgesetzt ist? Ich fürchte nein, und auch die Rente mit 67 erscheint mir für uns nicht realistisch, und zwar nicht einmal dann, wenn der Gesetzgeber endlich die Ungerechtigkeiten bei der Anerkennung der Kindererziehungszeiten beseitigen und die Mütterrenten unabhängig vom Geburtsdatum ihrer Kinder rechtlich gleich behandeln würde. In vielen Bereichen sind die Frauen den Männern nach wie vor nur auf dem Papier gleichgestellt. Unsere Kernforderungen entsprechen verblüffend oft sogar im Wortlaut dem, wofür unsere Vorkämpferinnen im djb, im Deutschen Juristinnen-Verein, im

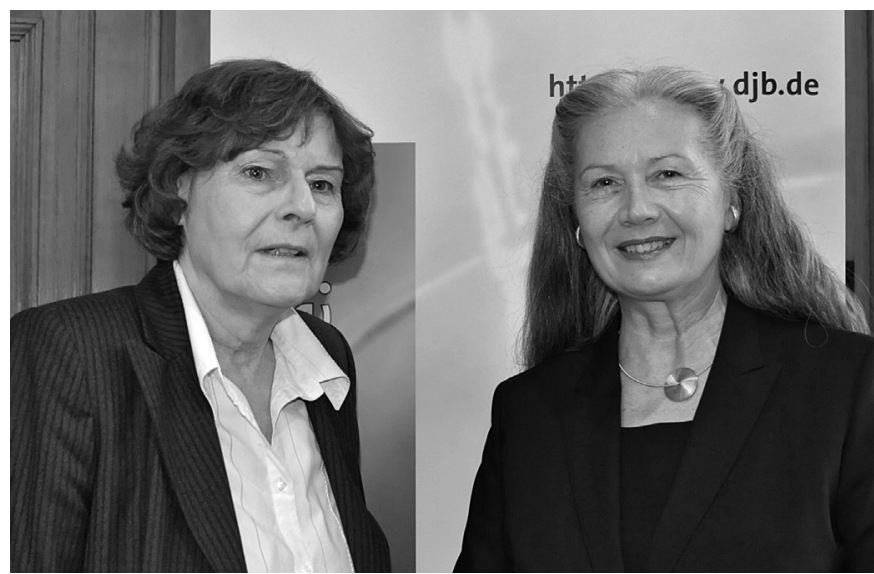

A Marion Eckertz-Höfer (Präsidentin des Bundesverwaltungsgerichts, Leipzig) und Ramona Pisal (Präsidentin des djb, Berlin).
ADF und den vielen anderen Frauenverbänden schon vor mehr als 100 Jahren gestritten haben. So ist Gewalt gegen Frauen und Kinder leider auch noch immer ein alltägliches Problem. Der djb arbeitet an vielen Baustellen - Sie erfahren mehr dazu auf unserer Homepage.

Zwar finden wir Frauen in verantwortlichen Positionen, mit Macht und Einfluss, mit ausreichendem Einkommen, von der Unternehmerin bis zur Universitätsrektorin, und es ist eine Frau, die vor wenigen Tagen mit einem überwältigenden Vertrauensbeweis der Wählerinnen und Wähler fast im Alleingang ihrer Partei die absolute Mehrheit im 18. Deutschen Bundestag gesichert hat. Aber es bleibt noch viel zu tun.

Unterstützen Sie uns, meine Herren, nehmen Sie Ihre zwei Vätermonate und noch einige mehr, nehmen Sie Teilzeit und Heimarbeit, Verzeihung: „home office“, in Anspruch, verweigern Sie Sitzungen nach 16 Uhr, bleiben Sie zu Hause, wenn das Kind krank ist, beantragen Sie Pflegezeit, und wir werden zusammen etwas bewegen, wenn ein Mann dasselbe Risiko für den Arbeitgeber bedeutet wie eine Frau.

Leipzig ist ein idealer Ort für einen solchen gemeinsamen Aufbruch, nicht nur wegen des ADF von 1865, wegen der fortschrittlichen Universität oder weil hier die Wahrheit über allem wacht, sondern vor allem darum, weil von Leipzig aus im Herbst 1989 schon einmal eine Welle ins Rollen gekommen ist, mit der niemand gerechnet hat und die dann unaufhaltsam wurde. Daran haben alle mitgewirkt, Frauen und Männer. Sie alle haben für ihren Wunsch nach einer gerechten und rechtsstaatlichen Politik viel riskiert, und am Ende gemeinsam sehr viel gewonnen, für uns alle. Nur darum können wir heute hier sein, und dafür sind wir den Leipzigern und den Leipzigerinnen unendlich dankbar.

Wir hören jetzt nach einem weiteren Stück von Franziska Hoelscher die Grußworte von Frau Dr. Grundmann, von Frau Munz und von Herrn Müller, dann gibt es noch einmal Musik und anschließend erwartet Sie die Festansprache von Frau Eckertz-Höfer.

Haben Sie vielen Dank für Ihre Aufmerksamkeit.

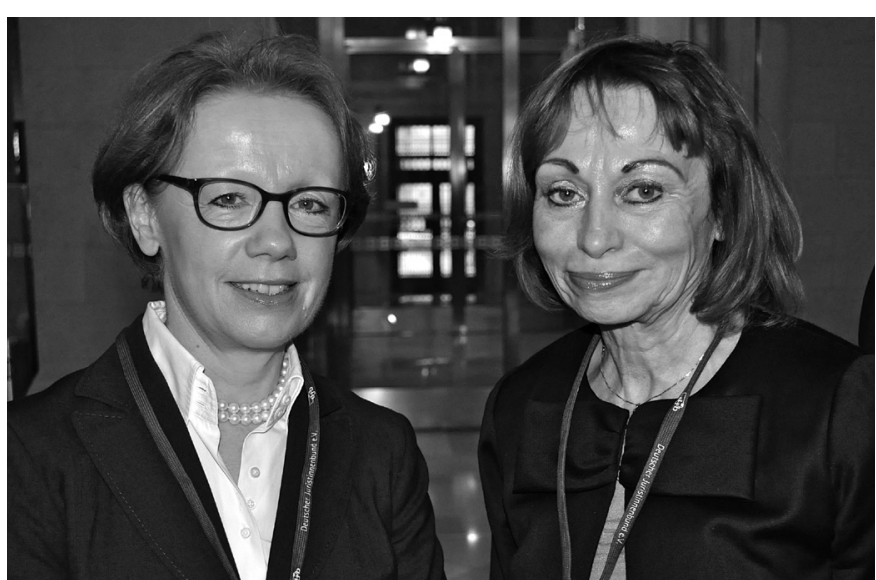

$\Delta$ Brigitte Meyer-Wehage (Direktorin des Amtsgerichts in Brake/Unterweser, Vorsitzende der djb-Kommission Zivil-, Familien- und Erbrecht) und Marie-Luise Graf-Schlicker (Abteilungsleiterin im Bundesministerium der Justiz, Berlin). 


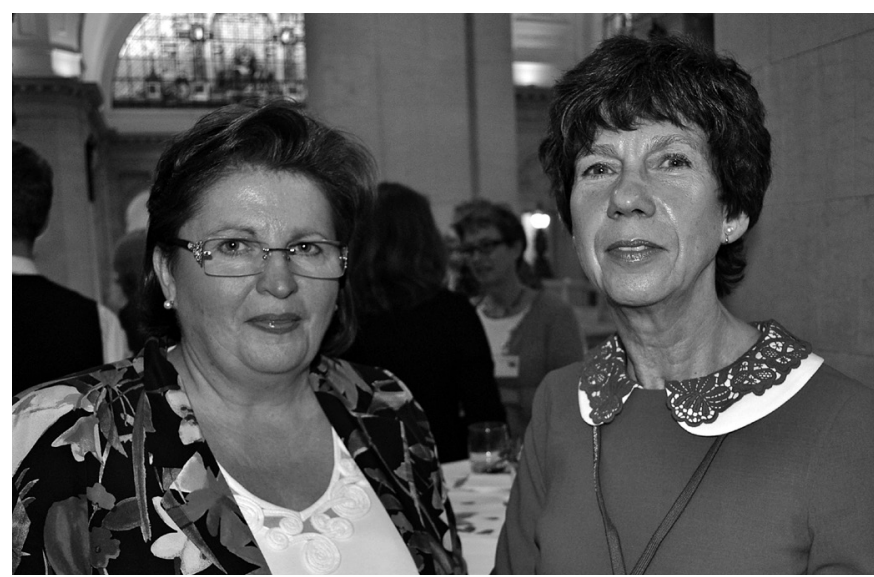

$\Delta$ Dr. Regina Vieler (Wirtschaftsprüferin und Steuerberaterin in Chemnitz, ehrenamtliche beisitzende Richterin beim BGH - Senat für Wirtschaftsprüfersachen), Birgit Munz (Präsidentin des Sächsischen Verfassungsgerichtshofs, Vizepräsidentin des Oberlandesgerichts, Dresden).

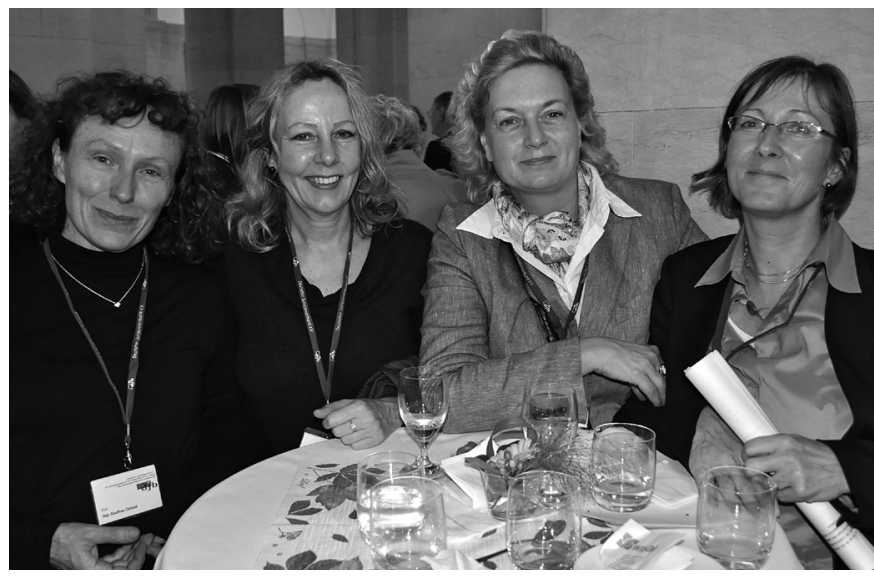

$\triangle$ Ruth Cohaus (Referentin im Ministerium der Finanzen des Landes SachsenAnhalt - Beteiligungsverwaltung, Magdeburg, djb-Kassenprüferin), Ruth Störtenbecker (Ministerialrätin, Staatskanzlei Sachsen-Anhalt, Magdeburg), Afra Waterkamp (Richterin am Finanzgericht, Magdeburg, Vorsitzende des djbLV Sachsen-Anhalt), Bettina Gärtner (Referentin im Ministerium für Arbeit und Soziales Sachsen-Anhalt, Magdeburg, Schatzmeisterin djb-LV Sachsen-Anhalt).

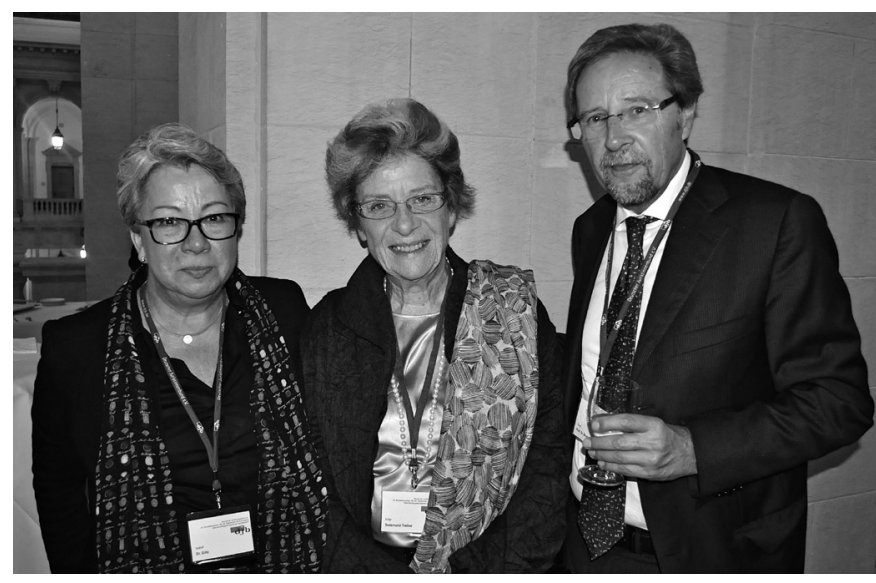

$\Delta$ Dr. Isabell Götz (Richterin am OLG, München, Vorsitzende Deutscher Familiengerichtstag - DFGT), Antje Sedemund-Treiber (Präsidentin des Bundespatentgerichts i.R., djb-Ehrenpräsidentin), Prof. Dr. Dr. h.c. Gerd Brudermüller (Vors. Richter am OLG, Karlsruhe, Ehrenvorsitzender DFGT).

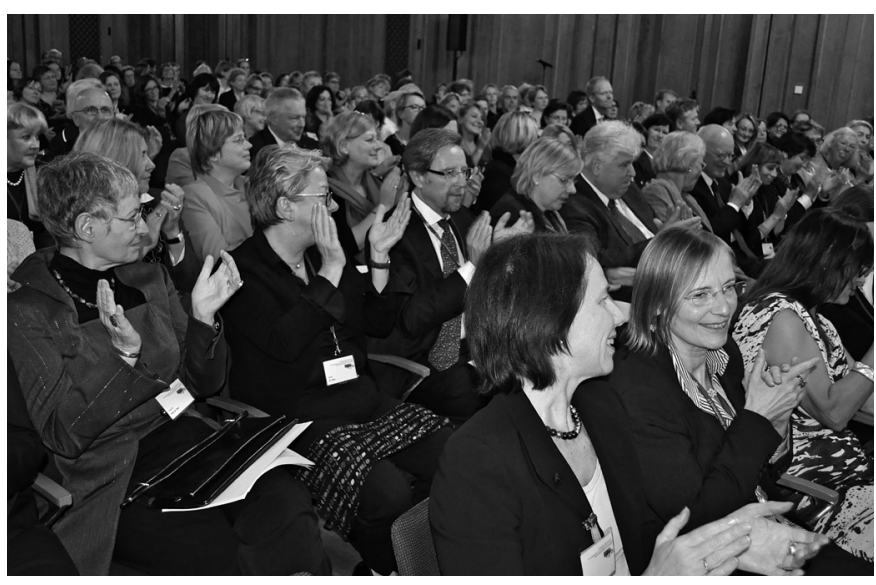

$\Delta$ Eröffnung des 40. djb-Bundeskongresses im Bundesverwaltungsgericht am 26. September 2013. Jeweils v.I.n.r. in der ersten Reihe: Elisabeth Kotthaus (Hauptverwaltungsrätin, Vertretung der Europäischen Kommission, Berlin, u.a. djb-Vizeppräsidentin 2003-2005), Margarete Hofmann (Direktorin, Europäische Kommission, Brüssel, djb-Vizepräsidentin), Mechtild Düsing (Rechtsanwältin, Münster, u.a. Vorstandsmitglied DAV). Zweite Reihe: Prof. Dr. Heide Pfarr (Senatorin a.D., Staatsministerin a.D., Direktorin des Wirtschafts- und Sozialwissenschaftlichen Instituts und Mitglied der Geschäftsführung der Hans-Böckler-Stiftung 1995-2011, Vorsitzende der djb-Kommission Arbeits-, Gleichstellungs- und Wirtschaftsrecht).

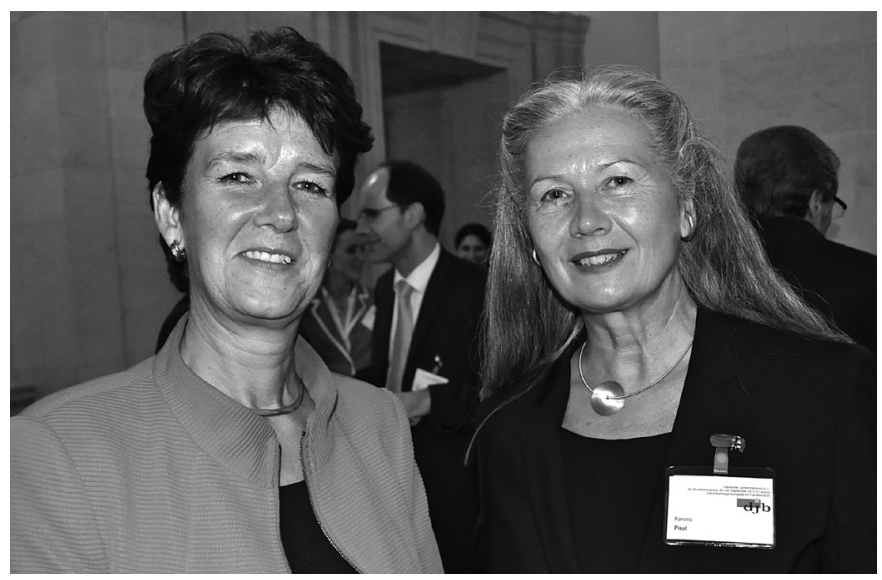

$\Delta$ Dr. Birgit Grundmann (Staatssekretärin des Bundesministeriums der Justiz, Berlin) und Ramona Pisal (Präsidentin des djb, Berlin).

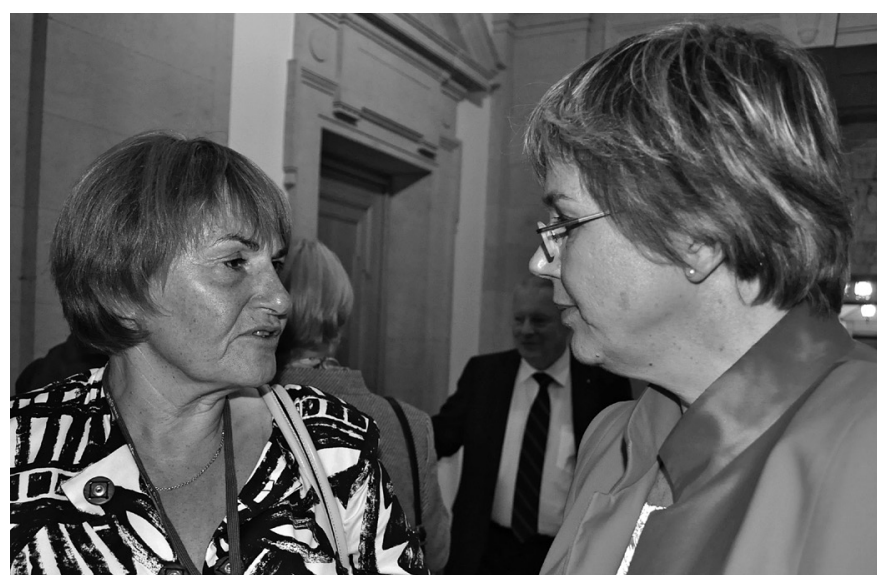

- Ingrid Schmidt (Präsidentin des Bundesarbeitsgerichts, Erfurt), Eva Maria Welskop-Deffaa (Ministerialdirektorin a.D., Bundesvorstand ver.di, Berlin). 


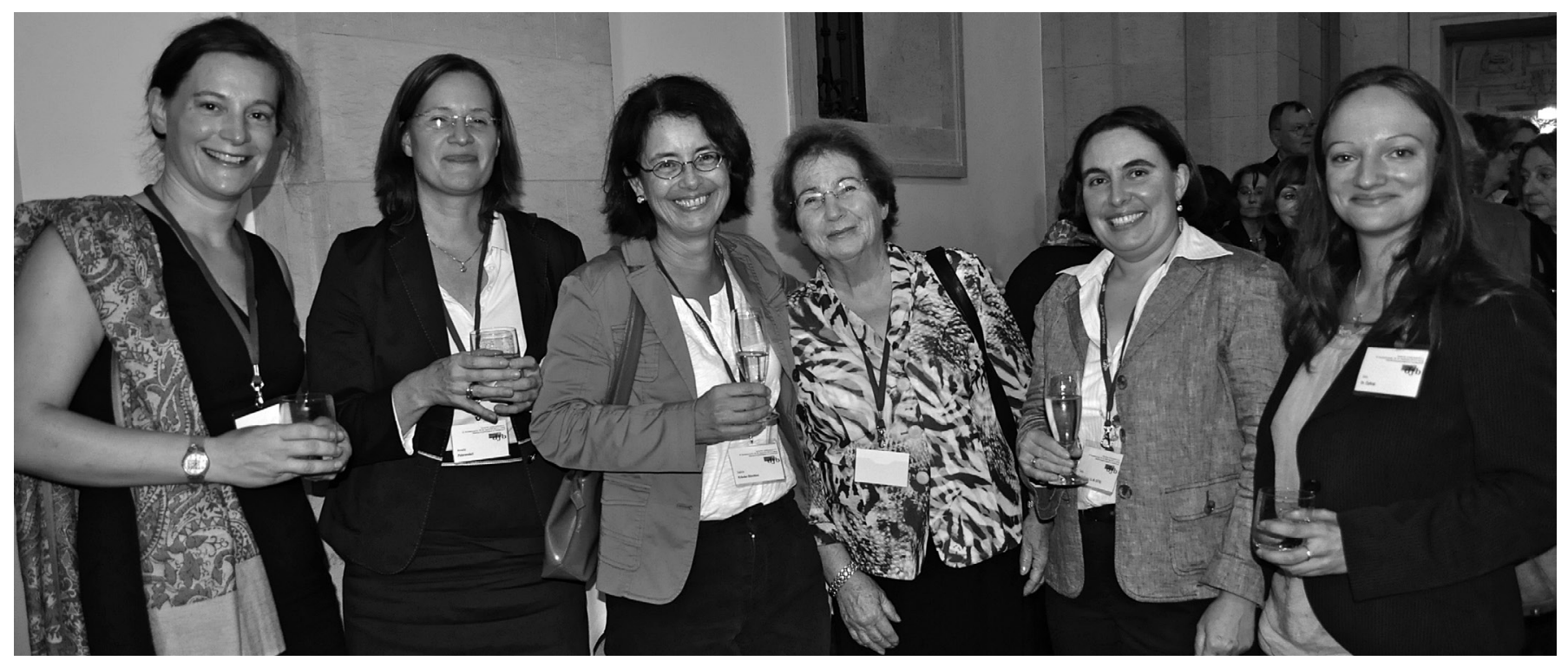

A Christiane Bergweiler (Rechtsanwältin in Pirmasens, Stellv. Vorsitzende des djb-LV Rheinland-Pfalz), Annette Peterandel (Rechtsanwältin in Saarbrücken, Stellv. Vorsitzende des djb-LV Saarland), Sabine Kräuter-Stockton (Oberstaatsanwältin, Saarbrücken, Vorsitzende des djb-LV Saarland), Christiane Schreiber (Rechtsanwältin, Saarbrücken, u.a. Vorsitzende der [Nichtsständigen] djb-Kommission Ältere Menschen 1991-2009), Tanja Fauth-Engel (Richterin am Amtsgericht Saarbrücken, Stellv. Vorsitzende und Schatzmeisterin des djb-LV Saarland), Dr. Sirin Özfirat (Richterin auf Probe, zur Zeit Ministerium der Justiz des Saarlandes).

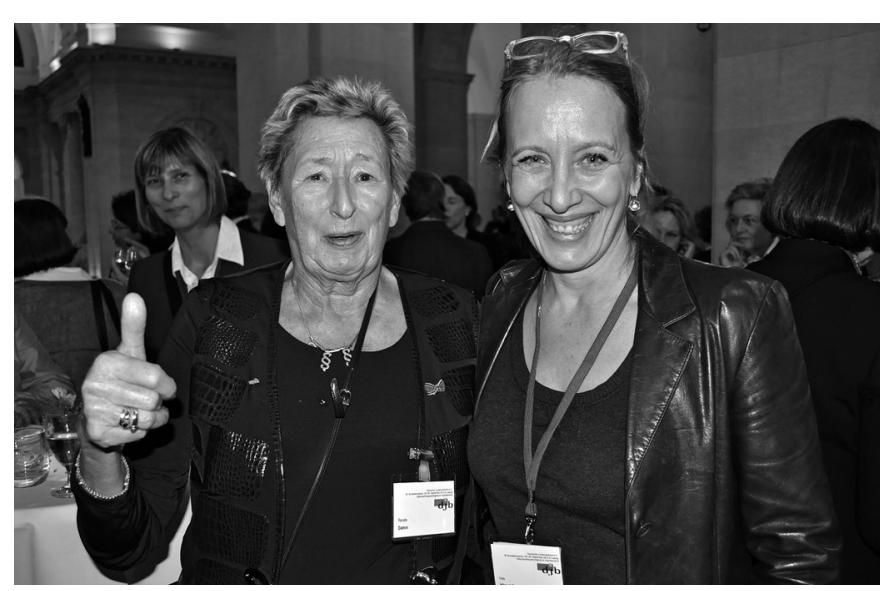

$\Delta$ Renate Damm (Rechtsanwältin in Hamburg, djb-Ehrenpräsidentin), Ines Hilpert-Kruck (Rechtsanwältin in Hamburg, Schriftführerin des djb-LV Hamburg).

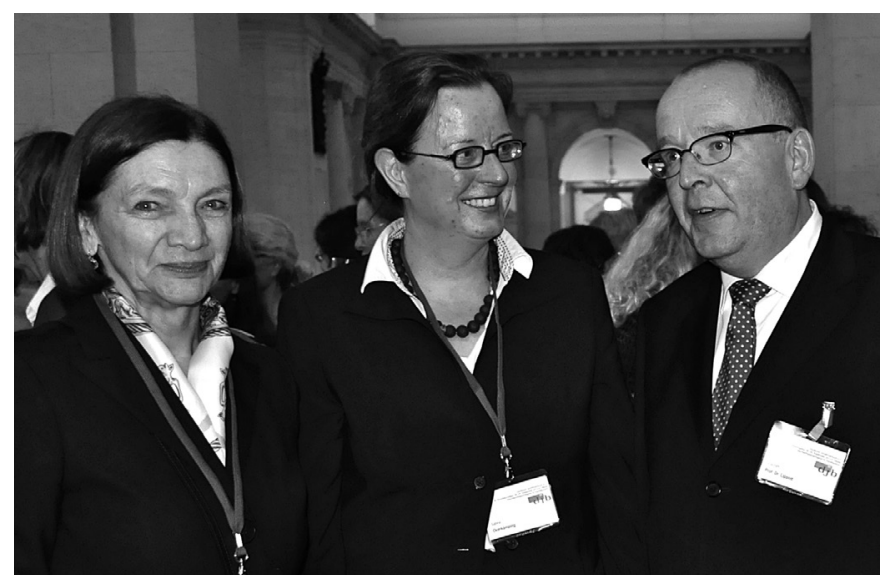

$\Delta$ Jutta Wagner (Rechtsanwältin und Notarin in Berlin, djb-Präsidentin 2005-2011), Sabine Overkämping (Ministerialrätin, Magdeburg, Vorsitzende der djb-Kommission Öffentliches Recht, Europa- und Völkerrecht), Prof. Dr. Joachim Lippott (Akademie Auswärtiger Dienst, Berlin).

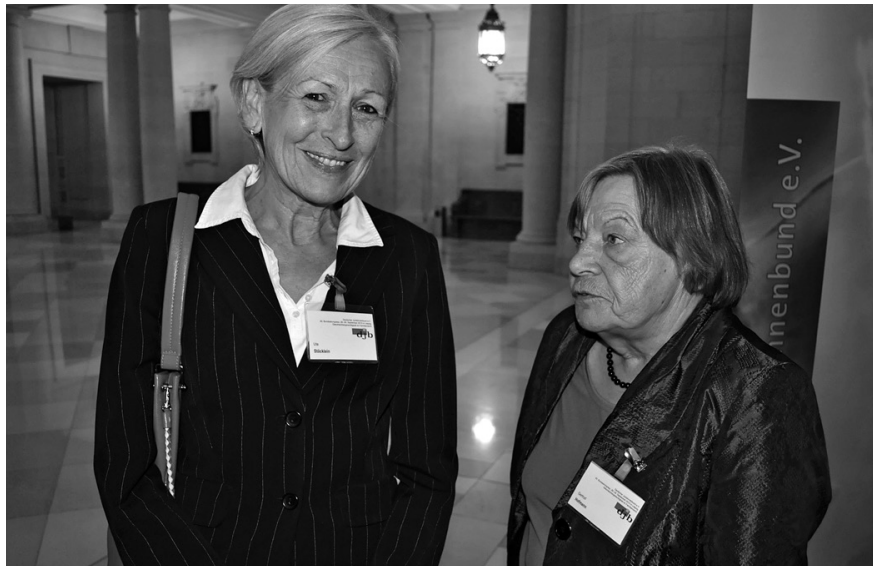

A Ute Stöcklein (Rechtsanwältin in Berlin) und Gertrud Hofmann (Vors. Richterin am OLG i.R., u.a. Ehrenvorsitzende der djb-RG München-Südbayern).

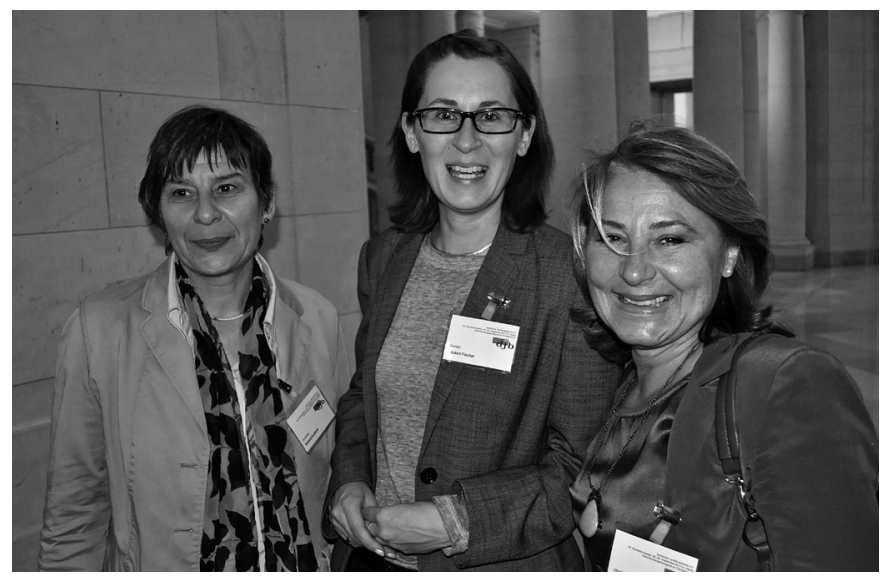

$\Delta$ Anneliese Schmid-Kaufhold (Rechtsanwältin in Freiburg, Vorsitzende der djb-RG Freiburg), Daniela Leukert-Fischer (Rechtsanwältin in Bensheim, Mitglied des djb-Bundesvorstands - Vorstand Regionalgruppenbeirat) und Jasmina Prpić LL.M, Vorsitzende Anwältinnen ohne Grenzen, Freiburg. 